\title{
Tumoral calcinosis producing peripheral nerve compression
}

\author{
A report of two cases \\ Ziv Williams, M.D., ${ }^{1,3}$ Kimberly K. Amrami, M.D, ${ }^{2}$ AND Robert J. SPINNER, M.D. ${ }^{1}$ \\ Departments of ${ }^{1}$ Neurosurgery and ${ }^{2}$ Radiology, Mayo Clinic, Rochester, Minnesota; and \\ ${ }^{3}$ Department of Neurosurgery, Massachusetts General Hospital, Boston, Massachusetts
}

\begin{abstract}
$\checkmark$ Tumoral calcinosis is a rare disorder that leads to diffuse calcium phosphate deposition into soft tissue and may be seen in the setting of uremia, hyperparathyroidism, or vitamin D intoxication. This lesion can produce significant local pain and can limit mobility in large joints where it tends to occur. Less commonly, it may produce neurological symptoms by compressing or encompassing adjacent neurovascular structures. Tumoral calcinosis involving nerve structures is challenging to treat, primarily because of its extensive size and propensity to infiltrate. Although surgical intervention can often provide symptomatic improvement, this lesion tends to recur in the presence of elevated calcium phosphate levels, and its management therefore requires a combined multidisciplinary surgical and medical approach. The authors describe two cases in which patients developed tumoral calcinosis producing peripheral nerve compression and discuss their respective surgical and medical management.
\end{abstract}

KEY WORDS - tumoral calcinosis - peripheral nerve compression

$\mathrm{T}$ UMORAL calcinosis is an uncommon benign condition in which soft-tissue structures and neighboring large joints can become extensively calcified. ${ }^{1,6,9}$ Tumoral calcinosis may be seen in the setting of primary or secondary hyperparathyroidism, vitamin D intoxication, and rare hereditary disorders of calcium metabolism..$^{8,10,11}$ Because of the increasing prevalence of chronic renal failure, this condition has also been more recently observed in the setting of hemodialysis. ${ }^{2,3}$ While the exact pathogenesis is not well understood, it appears that elevated serum levels of calcium and phosphate lead to deposition of insoluble calcium salts in soft tissue. This process can produce calcific tumoral lesions that may compress and occasionally envelop adjacent nerves. ${ }^{4,13,15}$ These tumors are often difficult to resect completely because of their size and propensity to infiltrate into surrounding soft tissue. They also frequently recur because of their diffuse nature and the difficulty of adequately controlling calcium phosphate levels. In the present study, we describe two such cases in which patients developed calcinotic lesions that produced peripheral nerve compression and discuss their management.

Abbreviations used in this paper: CT = computed tomography; $\mathrm{MR}=$ magnetic resonance.

\section{Case 1}

\section{Case Reports}

History and Presentation. This 31-year-old man had a 10year history of chronic renal failure due to adolescent reflux nephropathy. He had been treated with peritoneal dialysis for the past 5 years and had refused hemodialysis for reasons of personal preference. His mean calcium $\times$ phosphorus product averaged between 60 and $70 \mathrm{mg}^{2} / \mathrm{dL}^{2}$. The patient initially developed painful soft-tissue calcium deposits along the right elbow, which were sequentially followed by the development of similar lesions within the left shoulder, right ring finger and right shoulder over the course of 3 years.

Examination. On physical examination, the patient was found to have significant limitation of movement in the left shoulder, demonstrating a maximum range of approximately $60^{\circ}$ of active abduction and forward flexion, but his motor and sensory neurological function were found to be otherwise preserved. A radiograph of the left shoulder demonstrated extensive calcification around the shoulder and proximal humerus (Fig. 1A). A CT scan demonstrated a large calcific mass around the left shoulder between the coracoid process, clavicle, and proximal humerus and also 

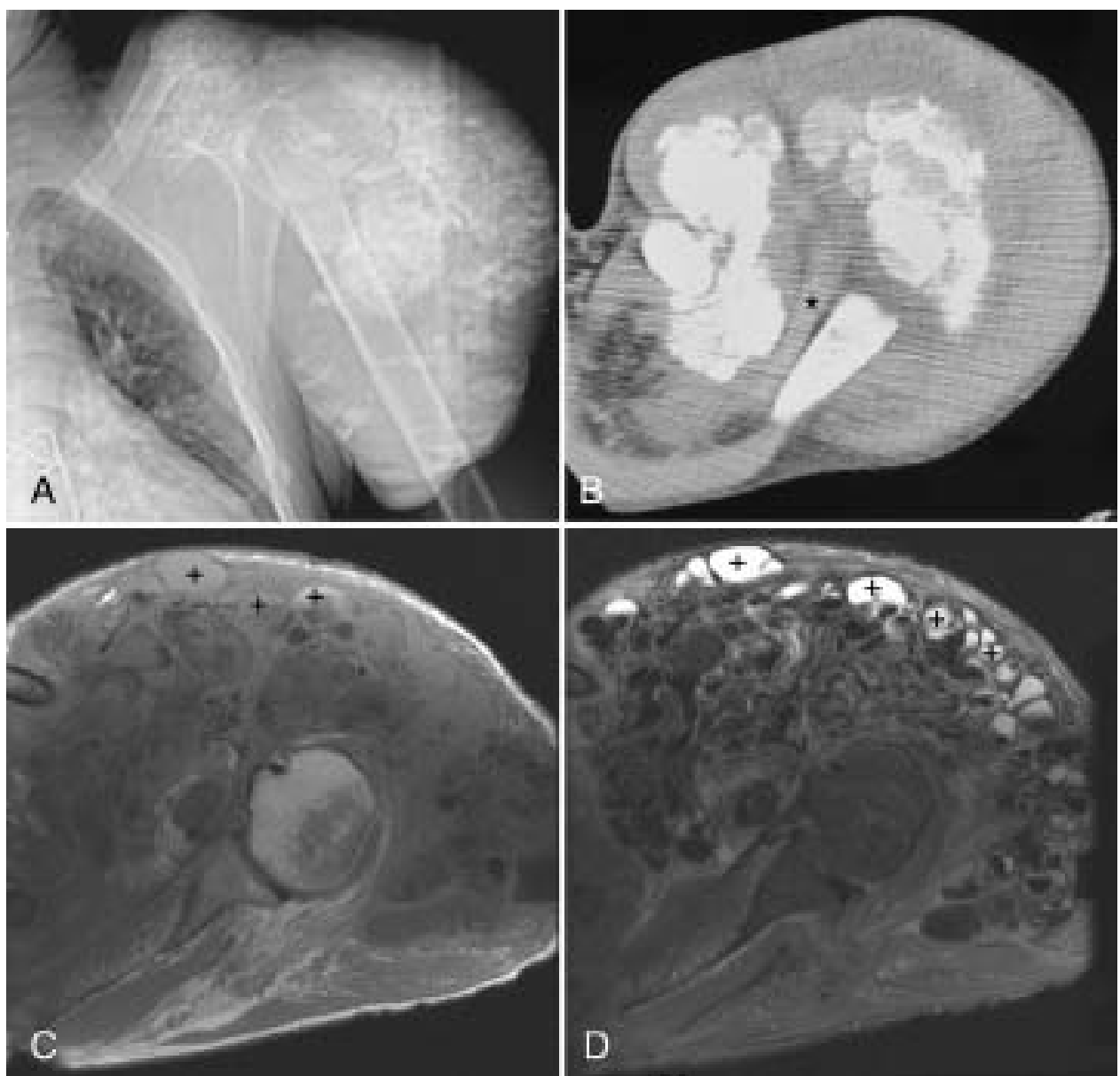

FIG. 1. Case 1. Preoperative images. A: Left anteroposterior oblique radiograph of the left shoulder and proximal humerus in internal rotation showing the extensive, amorphous calcification within the soft tissues surrounding the shoulder and upper arm. The underlying bone is unaffected. B: Axial CT scan of the left shoulder demonstrating the amorphous calcification within the supraclavicular fossa and surrounding the humeral head. There is associated mass effect on and distortion of the soft tissues especially in the region of the supraclavicular brachial plexus (asterisk). C: Axial T1-weighted fast-spin echo (FSE) MR image at the level of the glenohumeral joint shows a large, heterogeneous, softtissue abnormality around the shoulder, involving the pectoralis, deltoid, and coracobrachialis muscles. There are multiple loculated fluid spaces with fluid-fluid levels (plus signs) of varying signal intensity representing fluid and calcium in different states of ionization. The dependent fluid is dark in signal intensity, a finding compatible with calcium layering within the loculations. The underlying bone is not involved. D: Axial T2-weighted FSE MR image with fat suppression at the same level as figure $\mathrm{C}$ showing the extensive loculated fluid compartments within the soft tissues, with dependent dark calcium and superficial fluid within the loculations (plus signs).

showed mass effect on the supraclavicular brachial plexus, displacing it posteriorly (Fig. 1B). An MR imaging study demonstrated a complex soft-tissue mass around the shoulder with heterogeneous signal intensity on both T1- and T2weighted sequences. The brachial plexus was difficult to distinguish on MR imaging due to the associated extensive soft-tissue edema. There were multiple fluid spaces containing fluid-fluid levels with dependent hypointense material representing calcium. The appearance on all three modalities suggested tumoral calcinosis. The deltoid, pectoral, and coracobrachialis muscles (Fig. 1C and D) were all diffusely infiltrated by the lesion, but the underlying bones did not appear to be affected.

First Operation and Postoperative Course. In an attempt to prevent further progression of his tumoral calcinosis, the patient underwent a subtotal parathyroidectomy as well as an increase in the frequency of his dialysis. There was a transient reduction in his serum level of calcium phosphate, but the level subsequently increased. The patient's left shoulder lesion ultimately progressed to the point where it broke through the skin; the lesion was producing significant pain and diminishing mobility. Based on these findings, operative intervention was undertaken.

Second Operation. An anterior supra- and infraclavicular exposure was performed. The tumor was mobilized from underneath the deltoid and pectoralis major muscles inferiorly. In contrast to the superficial tumor component, which was easily mobilized, the mass infiltrated the posterior deltoid and latissimus muscles posteriorly, significantly limiting dissection. The mass had a chalky, fibrous appearance. Biopsy specimens demonstrated a large calcific mass with dense fibrous bands infiltrating the surrounding soft tissue. 
As dissection was carried inferiorly towards the quadrangular space, it appeared that the lesion was encompassing the axillary nerve. The tumor was gently dissected off the nerve, and neurological function was preserved as documented by muscle contraction with proximal nerve stimulation. Near complete resection was obtained.

Third Operation. Three years following the initial resection of the calcific mass, the patient returned with extensive skin breakdown over the lesion and advanced involvement of the tumoral calcinosis along the infraclavicular brachial plexus. Following reexposure of the brachial plexus through the prior incision, the tumor was again mobilized superficially and debulked (Fig. 2A and B). Dissection was carried down to the cords and terminal branches. Neurolysis of the musculocutaneous nerve up into the lateral cord was performed. The median nerve was also decompressed through development of a plane between the tumor and nerve. The remaining tumor, which was intimately adherent to the posterior aspect of the brachial plexus at the level of the clavicle, was left behind. The remaining overlying tumor was then resected in a piecemeal fashion. A nerve stimulator was used to confirm preservation of the musculocutaneous and median nerves through production of elbow flexion and finger flexion, respectively. Additional tumor was resected along the deltoid and pectoralis major and minor muscles, which were grossly infiltrated; the underlying neurovascular structures were protected.

Postoperative Course. The patient remained at his neurological baseline following surgery. Postoperative imaging demonstrated subtotal resection of the tumor with no residual compression of the adjacent neurovascular structures. Following surgery, the patient was treated with a maintenance regimen of frequent dialysis (five to six times a day), oral phosphate binding agents, and a diet low in calcium and phosphorus. He continued to refuse hemodialysis or consideration for renal transplantation.

\section{Case 2}

History and Presentation. This 32-year-old woman had a renal phosphate excretion deficiency of unknown etiology resulting in elevated phosphate levels in the setting of normal calcium levels. The patient had been well until the age of 12 when she first developed a calcific mass in her right hip, which caused significant pain and limited range of motion. Over the following 20 years, calcific masses had developed sequentially in her left hip, right shoulder, left shoulder, and both elbows. Extensive prior evaluation demonstrated mildly elevated 1,25-dihydroxy-vitamin D levels, but otherwise normal parathyroid hormone levels. She had previously undergone 10 surgeries to the right hip, two to the left hip, and three to the shoulders and elbows at other hospitals, primarily for removal of painful superficial soft-tissue calcific masses. The patient presented to our institution for treatment of increasing right thigh pain and limited range of hip motion.

Examination. On physical examination, the patient was found to have a firm, large, palpable soft-tissue mass in the right buttock. A CT scan of the pelvis demonstrated two large areas of amorphous calcification within the soft tissues of the buttock and thigh areas (Fig. 3A and B), with the right side affected more than the left. Similarly, MR im-

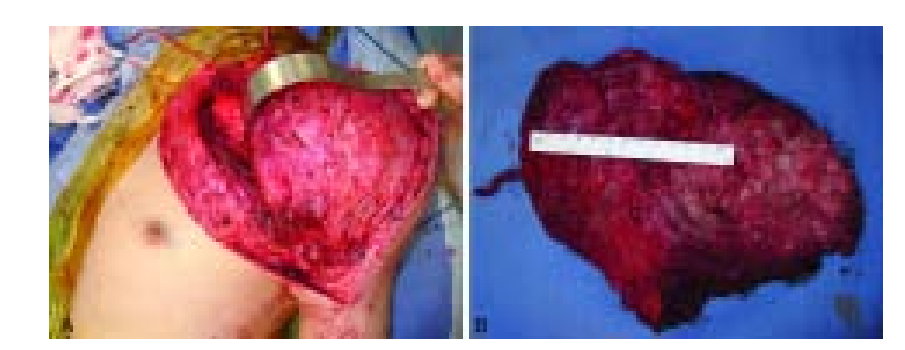

FIG. 2. Case 1. Intraoperative photograph (A) showing the lesion in situ and photograph (B) of the lesion after resection. The lesion was approximately $20 \times 16 \mathrm{~cm}$.

aging demonstrated large areas of hypointense but somewhat heterogeneous signal intensity on both $\mathrm{T} 1$ - and T2weighted sequences. The right-side lesion was producing significant mass effect on the sciatic nerve just distal to the notch, displacing it posteriorly. The sciatic nerve was hyperintense and slightly enlarged with associated denervation edema in the right gluteus musculature. The extensive calcifications involved the surrounding musculature of the right buttock and hip and the abductor muscles (Fig. 3C and D).

Operation. The lateral aspect of the thigh was incised revealing extensive tumoral calcinosis along the right trochanter, vastus lateralis, and abductor muscles. The sciatic nerve was identified, retracted medially, and protected throughout the procedure prior to resection of the tumor. The mass was displacing the nerve posteromedially but did not appear to be adherent to the nerve. The tumor was excised along the vastus lateralis and greater trochanter and the abductor muscles and posteriorly down to the ischium without difficulty. Histopathological examination of a tumor section demonstrated extensive soft-tissue calcium deposition with associated giant cells and surrounding reactive fibrosis.

Postoperative Course. Postoperative CT and MR imaging studies demonstrated gross-total resection of the tumor without evidence of residual compression of the sciatic nerve. The patient had no neurological deficit following surgery, but her postoperative course was complicated by a wound infection secondary to skin breakdown overlying the resection bed. The infection required serial irrigation and debridement of the wound. The patient continued to adhere to a low-phosphorous diet and to be treated with oral phosphate binding agents. Her phosphate levels were relatively well controlled at her most recent follow-up appointment. Given her considerable disease burden, she is being considered for renal transplantation.

\section{Discussion}

Although the exact pathogenesis of tumoral calcinosis is not well understood, the disorder appears to occur more frequently in patients with chronic renal failure and those treated with hemodialysis. ${ }^{2,3}$ Tumoral calcinosis has principally been described in the setting of abnormally elevated serum calcium phosphate levels and is associated with conditions such as hyperparathyroidism, osteolytic metastatic disease and vitamin D intoxication. ${ }^{8,10,11}$ Less extensive abnormal soft-tissue calcium deposits may also be seen in the setting of aluminum toxicity and advanced age. ${ }^{16}$ Tumoral calcinosis has also been described, albeit rarely, in the setting of 

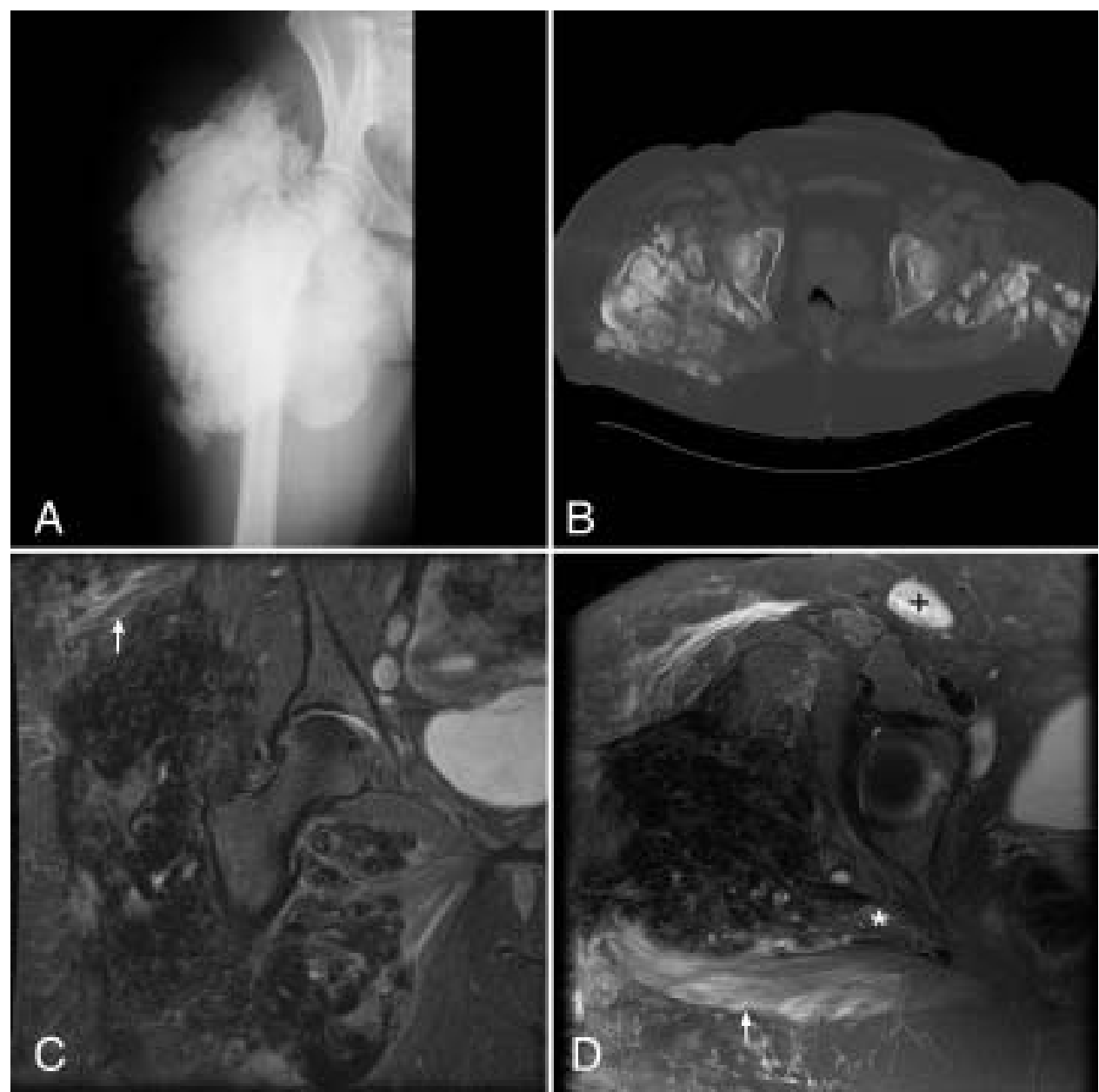

FIG. 3. Case 2. Preoperative images. A: An anteroposterior radiograph of the right hip showing extensive, amorphous calcifications surrounding the hip joint and extending into the gluteus musculature and proximal thigh muscles. B: Axial CT scan demonstrating the soft-tissue calcifications at both hips involving only the muscle. The process spares the underlying bone and adjacent subcutaneous tissues. C: Coronal T2-weighted FSE MR image with fat suppression of the right hip shows the hypointense material representing calcium surrounding the hip with some edema within the gluteus maximus muscle (arrow). D: Axial T2-weighted FSE MR image with fat suppression at the level of the sciatic notch demonstrates the extensive loculations within the muscles surrounding the hip. The sciatic nerve is hyperintense (asterisk) and displaced slightly posteriorly by the calcinotic lesion. There is associated denervation change in the gluteus muscle (arrow). Of note, there is an enlarged lymph node at the groin (plus sign), which is probably related to the underlying tumoral calcinosis inflammatory process.

isolated hyperphosphatemia, ${ }^{12}$ as in Case 2 in this article. Histologically, the lesions of tumoral calcinosis consist of lobulated calcific capsules within which are septated collagenous tissue fibers and foreign-body giant cells., ${ }^{913}$ The lesions may contain proteinaceous fluid as well as dense fluid concentrates of calcium phosphate and calcium carbonate. The lesions of tumoral calcinosis can be differentiated from regular ossification in that they lack the medullary trabecular pattern and organized cortical bone. Consistent with these histological findings, radiographs and CT scans show amorphous calcifications without chondroid characteristics or features of ossification. On MR images the lesions may have a loculated appearance with multiple fluid spaces or a more solid appearance, depending on the ionization state of the calcium in the lesion. Large lesions, in particular, will often show a fluid-fluid level or a "sedimentation sign," the result of dependent layering of the more hypointense calci- um. ${ }^{6,14,13}$ In the absence of a significant fluid component, there is relative low signal intensity on both $\mathrm{T} 1-$ and $\mathrm{T} 2-$ weighted sequences, which helps to distinguish the lesions of tumoral calcinosis form other lesions.

The clinical presentation of this lesion is primarily benign and commonly consists of pain, limited mobility in large joints, and skin breakdown. ${ }^{1}$ Less frequently, it may affect surrounding nerve structures leading to neurological deficit. Given the rarity of this disorder, only a few cases involving nerve structures have been reported in the literature thus far. $4,5,13,15$ Tumoral calcinosis with nerve involvement is difficult to treat, largely due to the extensive size of the lesions and their propensity to infiltrate surrounding soft tissue. The lesions are often expansive, occasionally eroding into bone, and may dramatically displace or encompass important neurovascular elements. In addition, there can be large soft tissue and skin defects after resection, and treatment may 
therefore require a combined surgical approach. In both cases presented here, the tumor extensively enveloped the neural elements in addition to infiltrating surrounding soft tissue and skin, and therefore necessitated the combined expertise of the neurosurgical, orthopedic, and plastic surgical teams.

Although surgical intervention can offer symptomatic improvement in many cases, postoperative medical management is essential for limiting tumor recurrence. Medical management includes prescribing a diet low in calcium, phosphorous, and protein, treating patients with phosphate binding medications, and maintaining strict control of metabolic acidosis..$^{1,911}$ Other measures, in the setting of uremia, include increasing the frequency of dialysis and lowering the concentration of calcium in the dialysate. ${ }^{2,3,16}$ Some authors have suggested that tumoral calcinosis may also benefit from resection of the parathyroid gland in the setting of hyperparathyroidism, ${ }^{8}$ but these measures are only recommended when both plasma calcium and parathyroid hormone levels are elevated. Although a subtotal parathyroidectomy was performed in Case 1 , the benefit of the procedure was short lasting. Lastly, renal transplantation has been advocated for cases of refractory tumoral calcinosis in which the patients' calcium phosphate levels remain persistently elevated and, in a few cases transplantation has resulted in dramatic remission of the disease.?

It has been suggested that the initial size of the lesions of tumoral calcinosis and presence of residual tumor are positive predictors of tumor recurrence. ${ }^{1}$ In both the cases presented in the current study, the tumors were large, firm, extensively infiltrated into soft tissue, and difficult to resect. Moreover, the lesions were enveloping or compressing major adjacent nerves, and the surgeons were required to identify and protect these structures early in the surgical procedures. Although complete resection is desirable, it is often unachievable. Close follow-up evaluation and continued medical treatment are therefore critical in the care of patients in these challenging cases.

\section{Acknowledgments}

We would like to thank Nho Tran for his invaluable contribution to the care of the patients whose cases are presented in this article.

\section{References}

1. Baums MH, Klinger HM, Otte S: Morbus Teutschlander-a massive soft-tissue calcification of the foot in a patient on long-term hemodialysis. Arch Orthop Trauma Surg 123:51-53, 2003
2. Fernández E, Amoedo ML, Borrás M, Pais B, Montoliu J: Tumoral calcinosis in haemodialysis patients without severe hyperparathyroidism. Nephrol Dial Transplant 8:1270-1273, 1993

3. Fernandez E, Montoliu J: Successful treatment of massive tumoral calcinosis with daily haemodialysis and very low calcium dialysate. Nephrol Dial Transplant 9:1207-1209, 1994

4. Garcia S, Cofan F, Combalia A, Campistol JM, Oppenheimer F, Ramon R: Compression of the ulnar nerve in Guyon's canal by uremic tumoral calcinosis. Arch Orthop Trauma Surg 120: 228-230, 2000

5. Garcia S, Cofan F, Combalia A, Casas A, Campistol JM, Oppenheimer F: [Compression of the sciatic nerve in uremic tumor calcinosis.] Neurologia 14:86-89 (Spn)

6. Geirnaerdt MJ, Kroon HM, van der Heul RO, Herfkens HF: Tumoral calcinosis. Skeletal Radiol 24:148-151, 1995

7. McGregor D, Burn J, Lynn K, Robson R: Rapid resolution of tumoral calcinosis after renal transplantation. Clin Nephrol 51: 54-58, 1999

8. Mockel G, Buttgereit F, Labs K, Perka C: Tumoral calcinosis revisited: pathophysiology and treatment. Rheumatol Int 25: 55-59, 2005

9. Pola E, Pola R, Gaetani E, De Santis E: Tumoral calcinosis. Lancet 359:1818, 2002

10. Quarles LD, Murphy G, Econs MJ, Martinez S, Lobaugh B, Lyles KW: Uremic tumoral calcinosis: preliminary observations suggesting an association with aberrant vitamin D homeostasis. Am J Kidney Dis 18:706-710, 1991

11. Reginato AJ, Tamesis E, Netter P: Familial and clinical aspects of calcium pyrophosphate deposition disease. Curr Rheumatol Rep 1:112-120, 1999

12. Savaci N, Avunduk MC, Tosun Z, Hosnuter M: Hyperphosphatemic tumoral calcinosis. Plast Reconstr Surg 105:162-165, 2000

13. Sharma M, Sinha R, Hussey K, Fouyas IP: Tumoral calcinosis of the filum terminale. Neurosurgery 57: E596, 2005

14. Steinbach LS, Johnston JO, Tepper EF, Honda GD, Martel W: Tumoral calcinosis: radiologic-pathologic correlation. Skeletal Radiol 24:573-578, 1995

15. Weiber H, Linell F: Tumoral calcinosis causing acute carpal tunnel syndrome. Case report. Scand J Plast Reconstr Surg Hand Surg 21:229-230, 1987

16. Zins B, Zingraff J, Basile C, Petitclerc T, Urena P, Bardin T, et al: Tumoral calcifications in hemodialysis patients: possible role of aluminium intoxication. Nephron 60:260-267, 1992

Manuscript submitted March 30, 2007.

Accepted April 26, 2007.

Address reprint requests to: Robert Spinner, M.D., Mayo Clinic, Gonda 8, 200 1st Street SW, Rochester, Minnesota 55902. email: Spinner.Robert@mayo.edu. 